\title{
Graphs of Permutation Groups
}

\author{
T. Chalapathi \\ Assistant Professor \\ Dept. of Mathematics \\ Sree Vidyanikethan Eng.College \\ Tirupati,-517502 \\ Andhra Pradesh, India
}

\author{
R. V. M. S. S. Kiran Kumar \\ Research Scholar \\ Dept.of Mathematics \\ S.V.University \\ Tirupati,-517502 \\ Andhra Pradesh, India
}

\begin{abstract}
In this paper we introduce and study permutation graphs of permutation groups. Basic, structural and specific properties of these graphs are investigated and characterized. Further, we obtain formulae for enumerating total number of shortest and longest cycles of permutation graphs.
\end{abstract}

\section{General Terms}

Algebraic Graph Theory, Group theory and Combinatorics.

\section{Keywords}

Permutation groups, Even and odd permutation graphs, Triangles, Hamilton cycles, Disjoint Hamilton cycles.

\section{INTRODUCTION}

The study of group theoretic graphs using the properties of graphs and groups has become incredible research topic in the recent years, and exiting to many fascinating results and questions. Graphs are called algebraic graphs if their constructions are based on modern algebraic structures and number theoretic functions, and graphs are called number theoretic graphs if their constructions are based on the number theoretic functions. Several mathematicians studied algebraic graphs on various algebraic structures, namely, semi-group, group, ring, field, vector space. By using and applying these inter disciplinary studies to obtain basic, structural and specific properties of many algebraic graphs.

Dejter and Giudici [1], Berrizabeitia and Giudici [2] and others have studied the cycle structure of graphs associated with certain number theoretic functions. Maheswari and Madhavi [3,4] studied the Hamilton cycles and triangles of the algebraic graphs associated with Euler totient function $\varphi(n) n \geq 1$, an integer and quadratic residues modulo a prime p. Chalapathi, Madhavi and Venkataramana [5] studied the enumeration of triangles in the algebraic graph associated with divisor function $d(n), n \geq 1$, an integer. Recently the authors Chalapathi and Kiran kumar [6] studied the structural properties of even free graphs of the group $Z_{2 n}$, which are group theoretic graphs under the correspondence of Combinatrics.

The name permutation graphs were first introduced in 1967 by Chartrand and Harary [7]. These graphs are group theoretic graphs, which are denoted by $G_{\sigma}$ whose vertex set is $\{1,2,3, \ldots, n\}$, and $x y$ is an edge of $G_{\sigma}$ if and only if $x>y$ and $\sigma^{-1}(x)<\sigma^{-1}(y) \quad$ or $\quad x<y$ and $\sigma^{-1}(x)>\sigma^{-1}(y)$. Here a permutation graph is a simple undirected associated with a permutation $\sigma$ in permutation group $S_{n}$. But we made a different definition of permutation graphs from the above, which are defined specifically on even and odd permutations of $S_{n}$. These are combinatorially interesting and it provides enumerating techniques for enumerating total number of cycles in these graphs. we have introduced even and odd permutation graphs associated with a permutation group $S_{n}$ for each $n \geq 3$. These graphs are inter related between two special branches of mathematics, namely, Group theory and Combinatorics. Algebraically, these graphs are notated by $G\left(S_{n}, A_{n}\right)$ and $G\left(S_{n}, B_{n}\right)$ which are disconnected and connected, and also complement graphs to each other respectively. Also we determine the basic, structural and some specific properties of these graphs. Further, we establish formulae for enumerating the total number of even and odd cycles of permutation graphs.

\section{BASIC PRELIMINARIES AND NOTATIONS}

In this paper basic definitions and concepts of graph theory are briefly presented. A graph $G$ consist of a nonempty set $V(G)$ of vertices and a set $E(G)$ of elements called edges together with a relation of a incidence which associates with each member a pair of vertices, called its ends. A graph with no loops and no multiple edges is called a simple graph whose order and size are $|V(G)|$ and $|E(G)|$ respectively.

For any vertex $x$ in a $\operatorname{graph} G, \operatorname{deg}(x)$ be the number of edges with the vertex $x$ as an end point. A graph in which all vertices have the same degree is called a regular graph. A graph $X$ is called connected if there is a path between any two distinct vertices in $G$. A graph $G$ is complete if every two distinct vertices in $G$ are adjacent. A complete graph with $n$ vertices is denoted by $K_{n}$. Also, a simple connected graph is Eulerian if and only if degree of its each vertex is even.

A permutation of $n$-labeled set $S$ is a function $f: S \rightarrow S$ that is both one-one and onto, here the function composition $O$ is a binary operation on the collection of all permutations of a set $S$. The group of all permutations of $S$ is the permutation group on $n$-labels and is denoted by $S_{n}$. Note that $S_{n}$ has $n$ ! elements and $S_{n}$ is non-ableain group for $n \geq 3$. Throughout the text, we consider $f \circ g=f g$ and $g o f=g f$. A permutation $f \in S_{n}$ is a cycle of length $k$, if there exist elements $a_{1}, a_{2}, a_{3}, \ldots, a_{k} \in S$ such that $f\left(a_{1}\right)=a_{2}, f\left(a_{2}\right)=a_{3}, \ldots, f\left(a_{k}\right)=a_{1}$ and $f(x)=x$ for all other elements $x \in S$. It is denoted by $f=\left(a_{1}, a_{2}, a_{3}, \ldots, a_{k}\right)$.The 
simplest permutation is a cycle of length 2 , which are called transpositions. We define a permutation in $S_{n}$ is said to be even if it can be expressed as an even number of transpositions. The collection of these even permutations forms a group, which is called an Alternating group on $n-$ labels, and it is denoted by $A_{n}$. Here $A_{n}$ is non-ablelian group for each $n \geq 4$ and $\left|A_{n}\right|=\frac{n !}{2}$. However, a permutation in $S_{n}$ is said to be odd if it can be expressed as an odd number of transpositions. The set of odd permutations in $S_{n}$ is not a group, and it is denoted by $B_{n}$. The set $B_{n}$ is non-empty subset of $S_{n}$, and $\left|B_{n}\right|=\frac{n !}{2}$. Further $A_{n} \cap B_{n}=\phi$ for each $n \geq 1$.

For further graph theoretic notations and terminology reader refer Harary [8] and for group theory we fallow Judson [9].

\section{EVEN PERMUTATION GRAPHS}

This section introduces even permutation graph of permutation group, and studied its properties.

Definition. 3.1 Let $n \geq 3$ be a positive integer, and let $A_{n}$ be the set of even permutations in $S_{n}$. Then the graph $G=(V, E)$ is called an even permutation graph whose vertex set is $V=S_{n}$ and edge set $E$ consisting of an unordered pair $(f, g)$ is an edge such that either $f g$ or $g f$ is an even permutation, and it is denoted by $G\left(S_{n}, A_{n}\right)$.

Example. 3.2 The Figure 3.2 shows the even permutation graph $G\left(S_{4}, A_{4}\right)$.



Fig.3.3. The even permutation graph $G\left(S_{4}, A_{4}\right)$.

The above example illustrates that the even permutation graph $G\left(S_{n}, A_{n}\right)$ is disconnected for each $n \geq 3$. These disconnected graphs contain exactly two complete components. This gives the following fundamental theorem for even permutation graphs.

\section{Fundamental Theorem. 3.4}

For each $n \geq 3$, the even permutation $\operatorname{graph} G\left(S_{n}, A_{n}\right)$ contains exactly two complete components.
Proof. Let $f, g$ be any two vertices in the graph $G\left(S_{n}, A_{n}\right)$. Then arise the following three cases on $f, g \in S_{n}$.

Case (i). When either $f \in A_{n}$ and $g \in B_{n}$ or $f \in B_{n}$ and $g \in A_{n}$. Since the product of even and odd or odd and even permutations must be odd. So, there is no edge between $f$ and $g$ in $G\left(S_{n}, A_{n}\right)$ This shows that $G\left(S_{n}, A_{n}\right)$ is disconnected.

Case (ii). If $f, g \in A_{n}$, then either $f g$ or $g f$ is again in $A_{n}$. So there exists an edge between the vertices $f$ and $g$ in $G\left(S_{n}, A_{n}\right)$. If $f_{1}, f_{2}, f_{3}, \ldots, f_{\frac{n}{2}}$ are in $A_{n}$, then there exists an edge between any two vertices of these vertices, and hence the set of vertices $f_{1}, f_{2}, f_{3}, \ldots, f_{\frac{n !}{2}}$ form a complete sub-graph, which is a component of $G\left(S_{n}, A_{n}\right)$.

Case (iii). If $f, g \in B_{n}$, then either $f g$ or $g f$ is in $A_{n}$.So, in this case also, there exists an edge between the vertices $f$ and $g$ in $G\left(S_{n}, A_{n}\right)$.Suppose the vertices $g_{1}, g_{2}, g_{3}, \ldots, g_{\frac{n}{2}}$ are in $B_{n}$,then there exists an edge between any two of these vertices and hence the set of vertices $g_{1}, g_{2}, g_{3}, \ldots, g_{\frac{n !}{2}}$ form a complete graph, which is another component of $G\left(S_{n}, A_{n}\right)$.

From Case (i), Case (ii) and Case (iii), we obtain $G\left(S_{n}, A_{n}\right)$ disconnected, and hence it contains exactly two complete components.

Remark. 3.5 The two components of $G\left(S_{n}, A_{n}\right)$ denoted by $G\left(A_{n}, A_{n}\right)$ and $G\left(B_{n}, A_{n}\right)$ which are both complete. The fundamental theorem of the graph $G\left(S_{n}, A_{n}\right)$ immediate provides the following consequences, but their proofs are obviously trivial from the basic principles of combinatorial number theory [10].

1. For each $n \geq 3,\left|G\left(A_{n}, A_{n}\right)\right|=\left|G\left(B_{n}, A_{n}\right)\right|=\frac{n !}{2}$.

2. For each $n \geq 3$, the size of each component is $\left(\begin{array}{c}n ! / 2 \\ 2\end{array}\right)$ edges.

3. Each component of even permutation graph is not bipartite.

Theorem. 3.6 For each $n \geq 3$, we have $G\left(A_{n}, A_{n}\right)$ $\cong G\left(B_{n}, A_{n}\right)$.

Proof: Define a map $\varphi$ from the component $G\left(A_{n}, A_{n}\right)$ to the component $G\left(B_{n}, A_{n}\right)$ by the relation $\varphi(f)=g$, for every $f \in A_{n}$. We observe that $\varphi$ is one-to-one and onto, because $\varphi$ has an inverse function $\varphi^{-1}$ defined by $\varphi^{-1}(g)=f$, for each $g \in B_{n}$. It can be checked that $\varphi$ preserves adjacency, in the following sense: Let $f, f^{\prime}, g, g^{\prime} \in S_{n}$. Then, clearly, assume that $f, f^{\prime} \in A_{n}$ and $g, g^{\prime} \in B_{n}$. Therefore, $f f^{\prime} \in A_{n}$ if and only if $g g^{\prime} \in A_{n}$. It is evident that for each edge $\left(f, f^{\prime}\right)$ in $G\left(A_{n}, A_{n}\right)$ there exist an edge $\left(g, g^{\prime}\right)$ in $G\left(B_{n}, A_{n}\right)$ under the 
bijective map $\varphi$. That is, the map $\varphi$ preserves the adjacency. Hence $G\left(A_{n}, A_{n}\right) \cong G\left(B_{n}, A_{n}\right)$, for each $n \geq 3$.

Example. 3.7 The following figure shows that the components $G\left(A_{3}, A_{3}\right)$ and $\quad G\left(B_{3}, A_{3}\right)$ of $\quad G\left(S_{3}, A_{3}\right)$ are isomorphic since there is a graph isomorphism $\varphi: G\left(A_{3}, A_{3}\right) \rightarrow G\left(B_{3}, A_{3}\right)$ such that $\varphi\left(f_{1}\right)=g_{1} \quad, \quad \varphi\left(f_{2}\right)=g_{2}$ and $\varphi\left(f_{3}\right)=g_{3}$.

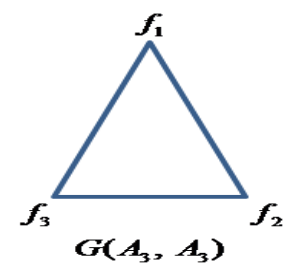

$\cong$

$G\left(A_{3}, A_{3}\right)$

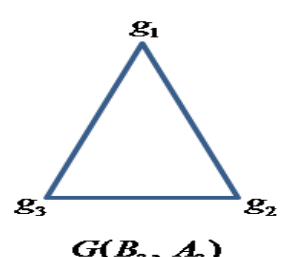

$G\left(B_{3}, A_{3}\right)$
Figure 3.8. The components $G\left(A_{3}, A_{3}\right)$ and $G\left(B_{3}, A_{3}\right)$ are isomorphic.

\section{ODD PERMUTATION GRAPHS}

In the above section, we constructed disconnected graphs $G\left(S_{n}, A_{n}\right)$ for each $n \geq 3$, and studied their properties. Due to this reason, we construct the complement of the graphs $G\left(S_{n}, A_{n}\right)$, and study its basic and structural properties. In this manner, the vertex set of complement graph is same as $S_{n}$ and whose edges are the pairs of non-adjacent vertices of $G\left(S_{n}, A_{n}\right)$. The notation and construction of these complement graphs are immediate fallows.

Definition. 4.1 For each $n \geq 3$, the graph $G\left(S_{n}, B_{n}\right)$ is called an odd permutation graph whose vertex set is $S_{n}$ and for each $f, g \in S_{n}$, the edge set is treated as $E=\left\{(f, g)\right.$ : either $f g$ or $\left.g f \in B_{n}\right\}$.

Example. 4.2 The Figure [4.3] show that the odd permutation graph of the permutation group $S_{4}$.

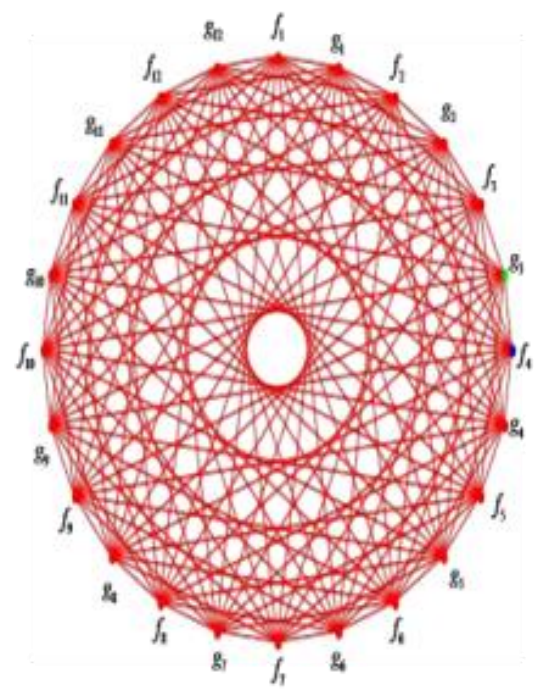

Fig.4.3. The odd permutation graph $G\left(S_{4}, B_{4}\right)$.

The odd permutation graph shows that the following basic properties.
1. The total number of vertices and edges of $G\left(S_{n}, B_{n}\right)$ are $n !$ and $\left(\frac{n !}{2}\right)^{2}$ respectively.

2. The degree of each vertex of $G\left(S_{n}, B_{n}\right)$ is $\frac{n !}{2}$. In particular, $G\left(S_{n}, B_{n}\right)$ is $\frac{n !}{2}$-regular.

3. The graph $G\left(S_{n}, B_{n}\right)$ is connected and regular but not complete.

Theorem.4.4 The graph $G\left(S_{n}, B_{n}\right)$ is Eulerian if $n \neq 3$.

Proof: Since $\frac{n !}{2}$ is odd if and only if $n=3$. For this reason, we consider $n \neq 3$. In this case $\frac{n !}{2}$ must be even for each $n>3$. This shows that the degree of each vertex in $G\left(S_{n}, B_{n}\right)$ is even, and hence $G\left(S_{n}, B_{n}\right)$ is Eulerian.

Definition. 4.5 A simple graph is self-complementary if it is isomorphic to its complement. Every self-complementary graph is connected but converse need not be true.

Theorem. 4.6 The graph $G\left(S_{n}, B_{n}\right)$ is not selfcomplementary.

Proof. If possible assume that $G\left(S_{n}, B_{n}\right)$ is selfcomplementary, then $n !=0,1(\bmod 4)$. This implies that $4|(n !-0), 4|(n !-1)$, which is a contradiction to the fact that $4+3$ ! and $4+(n !-1)$ for each $n \geq 3$. So, our assumption is wrong and hence $G\left(S_{n}, B_{n}\right)$ is not self-complementary graph.

A connected graph in which every vertex has degree 2 is called cycle. It is denoted by $C_{n}$ where $n$ is the number of vertices. If $n$ is even then $C_{n}$ is called an even cycle, and if $n$ is odd then $C_{n}$ is called an odd cycle. In $C_{n}$, the number of edges coincides with the number of vertices, and it is called the length of the cycle. In particular, the cycle $C_{3}$ is called a triangle, which is a shortest cycle. In simple undirected graphs, if there are cycles then they must have length at least three.

\section{ENUMERATION OF TRIANGLES IN $G\left(S_{n}, B_{n}\right)$}

In this section we enumerate the total number of triangles in the odd permutation graph, and hence deduce that the total number of odd cycles. The following theorem illustrates the enumeration of triangles in $G\left(S_{n}, B_{n}\right)$.

Theorem. 5.1 For each $n \geq 3$, the total number of triangles in the graph $G\left(S_{n}, B_{n}\right)$ is zero.

Proof. Let $f, g, h$ be any three vertices of the graph $G\left(S_{n}, B_{n}\right)$.Then the unordered pairs $(f, g),(g, h)$ and $(h, f)$ are edges in $G\left(S_{n}, B_{n}\right)$ if $f g$, ghand $h f$ are in $B_{n}$ for each $n \geq 3$. We show that the total number of triangles in $G\left(S_{n}, B_{n}\right)$ is zero. If possible assume that there exists a triangle $C_{3}=(f, g, h, f)$ in $G\left(S_{n}, B_{n}\right)$, then the pairs 
$(f, g),(g, h)$ and $(h, f)$ are edges of $G\left(S_{n}, B_{n}\right)$, that is, $f g, g h$ and $h f$ are in $B_{n}$. Now arise the following two cases.

Case (i). Suppose $f, g$ and $h$ are all either even or odd permutations then clearly $f g, g h$, and $h f$ are not in $B_{n}$.

Case (ii). If any two of $f, g$ and $h$ are either even or odd permutations then at least one of the product $f g, g h$ and $h f$ is not in $B_{n}$.

From Case (i) and Case (ii), our assumption is not true. This shows that the total number of triangles in odd permutation graph $G\left(S_{n}, B_{n}\right)$ is zero.

Corollary. 5.2 For each $n \geq 3$, the total number of odd cycles in $G\left(S_{n}, B_{n}\right)$ is zero.

Proof. Follows from Theorem 5.1.

We observe that, if $k$ is odd then $G\left(S_{n}, B_{n}\right)$ does not contain an odd cycle. It gives $G\left(S_{n}, B_{n}\right)$ is a bipartite graph, because there is a special relation between bipartite graphs and their cycles, and it states that a graph is bipartite if and only if it has no odd cycles [11]. So, the bipartite graphs are characterized by the absence of cycles of odd length.

Theorem. 5.3 For each $n \geq 3$, the odd permutation graph $G\left(S_{n}, B_{n}\right)$ is complete bipartite.

Proof. Let $V$ be the vertex set of odd permutation graph $G\left(S_{n}, B_{n}\right)$. Then $V=S_{n}$. This vertex set can be partitioned into two disjoint sets $V_{1}$ and $V_{2}$ of $V$ such that $V_{1}=\left\{f \in S_{n}: f \quad\right.$ is even $\}$ and $V_{2}=\left\{f \in S_{n}: f\right.$ is odd $\}$. Here $V_{1}$ and $V_{2}$ are called parts of the graph $G\left(S_{n}, B_{n}\right)$ in which every vertex from part $V_{1}$ is adjacent to every vertex from part $V_{2}$. Hence $G\left(S_{n}, B_{n}\right)$ is a complete bipartite graph.

Definition. 5.4 A simple undirected graph is called a triangle free graph if it contains no triangles.

From Theorem 5.1, the odd permutation graph $G\left(S_{n}, B_{n}\right)$ is a triangle free graph.

\section{ENUMERATION OF EVEN CYCLES}

\section{IN $G\left(S_{n}, B_{n}\right)$}

In this section, we describe the formula for enumerating the total number of even cycles in $G\left(S_{n}, B_{n}\right)$ for each $n \geq 3$. For this we immediate state the multiplication and addition principles in the Combinatorics.

Theorem. 6.1 [10] Suppose a procedure can be broken into $m$ successive stages, with $r_{1}$ different out comes in the first stage, $r_{2}$ different outcomes in the second stage, $\ldots$, and $r_{m}$ different out comes in the $m^{\text {th }}$ stage. If the number of outcomes at each stage is independent of the choices in previous stages, then the total procedure has $r_{1} r_{2} \ldots r_{m}$ different composite out comes.
Theorem. 6.2 [10] If there are $r_{1}$ different objects in the first set, $r_{2}$ different objects in the second set,..., $r_{m}$ different in the $m^{\text {th }}$ set, and if the different sets are disjoint, then the number of ways to select an object from one of the $m$ sets is $r_{1}+r_{2}+\ldots .+r_{m}$.

Theorem. 6.3 Let $k$ be an even positive integer such that $3<k \leq n$ !, $n \geq 3$. Then the total number of $k$-cycles in $G\left(S_{n}, B_{n}\right)$ is $\left(\begin{array}{l}n ! / 2 \\ k / 2\end{array}\right)^{2}$.

Proof. Let $n \geq 3$ be a positive integer and let $k$ be an even positive integer such that $3<k \leq n !$. Then the $k$-cycles in the graph $G\left(S_{n}, B_{n}\right)$ is either $\left(f_{1}, g_{1}, f_{2}, g_{2}, \ldots, g_{k}, f_{1}\right)$ or $\left(g_{1}, f_{1}, g_{2}, f_{2}, \ldots, f_{k}, g_{1}\right)$. Either of these two cycles shows that between any two odd permutations there exists an even permutation and vice versa. Since $k \geq 4$ is an even integer. It shows that $\frac{k}{2}$ is either even or odd integer. So that the $k-$ cycle in the graph $G\left(S_{n}, B_{n}\right)$ contains $\frac{k}{2}$ even and $\frac{k}{2}$ odd permutations. Thus the number of arrangements of $f_{1}, f_{2}, \ldots, f_{\frac{k}{2}}$ odd permutations are arranged between different fixed $g_{1}, g_{2}, \ldots, g_{\frac{k}{2}}$ even permutations from a collection of $\frac{n !}{2}$ permutations in $S_{n}$ is equal to $\left(\begin{array}{l}n ! / 2 \\ k / 2\end{array}\right)$ and thus each of these arrangement form a $k\left(=\frac{k}{2}+\frac{k}{2}\right)$-cycle in the $G\left(S_{n}, B_{n}\right)$. From Theorem [6.1], the total number of $k$-cycles in the graph $G\left(S_{n}, B_{n}\right)$ is $\left(\begin{array}{l}n ! / 2 \\ k / 2\end{array}\right)\left(\begin{array}{l}n ! / 2 \\ k / 2\end{array}\right)=\left(\begin{array}{l}n ! / 2 \\ k / 2\end{array}\right)^{2}$.

Example. 6.4 The following table illustrates the graphs $G\left(S_{3}, B_{3}\right)$ and $G\left(S_{4}, B_{4}\right)$, and their corresponding $k$-cycles. 


\begin{tabular}{|c|c|}
\hline Graphs & $k-$ cycles \\
\hline$G\left(S_{3}, B_{3}\right)$ & $4-$ cycles $=\left(\begin{array}{l}3 \\
2\end{array}\right)^{2}$ \\
\hline \multirow{11}{*}{$G\left(S_{4}, B_{4}\right)$} & $4-$ cycles $=\left(\begin{array}{l}12 \\
2\end{array}\right)^{2}$ \\
\hline & $6-$ cycles $=\left(\begin{array}{l}12 \\
3\end{array}\right)^{2}$ \\
\hline & $8-$ cycles $=\left(\begin{array}{l}12 \\
4\end{array}\right)^{2}$ \\
\hline & $10-$ cycles $=\left(\begin{array}{l}12 \\
5\end{array}\right)^{2}$ \\
\hline & $12-$ cycles $=\left(\begin{array}{l}12 \\
6\end{array}\right)^{2}$ \\
\hline & $14-$ cycles $=\left(\begin{array}{l}12 \\
7\end{array}\right)^{2}$ \\
\hline & $16-$ cycles $=\left(\begin{array}{l}12 \\
8\end{array}\right)^{2}$ \\
\hline & $18-$ cycles $=\left(\begin{array}{l}12 \\
9\end{array}\right)^{2}$ \\
\hline & $20-$ cycles $=\left(\begin{array}{l}12 \\
10\end{array}\right)^{2}$ \\
\hline & $22-$ cycles $=\left(\begin{array}{l}12 \\
11\end{array}\right)^{2}$ \\
\hline & $24-$ cycles $=\left(\begin{array}{l}12 \\
12\end{array}\right)^{2}$ \\
\hline
\end{tabular}

Definition. 6.5 Let $G$ be a simple undirected graph, then the girth of $G$ is the length of a shortest cycle in $G$, and it is denoted by $\operatorname{gir}(G)$.

Theorem. 6.6 The girth of an odd permutation graph $G\left(S_{n}, B_{n}\right)$ is 4 .

Proof. We know that the graph $G\left(S_{n}, B_{n}\right)$ is triangle free graph, therefore,

$$
\operatorname{gir}\left(G\left(S_{n}, B_{n}\right)\right) \neq 3 \Rightarrow \operatorname{gir}\left(G\left(S_{n}, B_{n}\right)\right) \geq 4 .
$$

Further, the graph $G\left(S_{n}, B_{n}\right)$ has a cycle of the form $C_{4}=\left(f_{i}, g_{i}, f_{j}, g_{j}, f_{i}\right)$ of

length 4 , which is smallest. Hence $\operatorname{gir}\left(G\left(S_{n}, B_{n}\right)\right)=4$. This completes the proof.

The diameter of a simple graph $G$, denoted by $\operatorname{diam}(G)$, is given by $\operatorname{diam}(G)=\max \{d(x, y): x, y \in V(G)\}$, where $d(x, y)$ is the length of the shortest path from $x$ to $y$.
Theorem.6.7 The diameter of odd permutation graph is 2 .

Proof: Consider the odd permutation graph $G\left(S_{n}, B_{n}\right), n \geq 3$ having the vertices of the form $f_{1}, f_{2}, \ldots, f_{\frac{n !}{2}}, g_{1}, g_{2}, \ldots, g_{\frac{n !}{2}}$. Since the vertex $f_{i}$ is not adjacent to $f_{j}$ and similarly the vertex $g_{i}$ is not adjacent to $g_{j}$ for every $1 \leq i, j \leq \frac{n !}{2}$. It is clear that there exist an edge between $f_{i}$ and $g_{i}$ or $f_{i}$ and $g_{j}$ because $f_{i} g_{i}, f_{i} g_{j} \in B_{n}$, so, $d\left(f_{i}, g_{i}\right)=1, d\left(f_{i}, g_{j}\right)=1$, $d\left(f_{i}, f_{j}\right)>1$ and $d\left(g_{i}, g_{j}\right)>1$, but in the graph $G\left(S_{n}, B_{n}\right)$, $n \geq 3$, there always exist either a path $f_{i}-g_{i}-f_{j}$ or $g_{i}-f_{i}-g_{j}$ for each $1 \leq i, j \leq \frac{n !}{2}$, which gives $d\left(f_{i}, f_{j}\right)=2$ and $d\left(g_{i}, g_{j}\right)=2$, for every $i \neq j$. It follows that $\operatorname{diam}\left(G\left(S_{n}, B_{n}\right)\right)=2$.

\section{ENUMERATION OF HAMILTON CYCLES IN $G\left(S_{n}, B_{n}\right)$}

In this section we study the Hamiltonian property of odd permutation graphs, and also establish a formula for enumerating total number of Hamilton cycles and their corresponding disjoint Hamilton cycles in the odd permutation graphs.

A Hamilton cycle in a simple undirected graph $G$ is a cycle containing every vertex of $G$, and $G$ is called a Hamilton graph if it contains a Hamilton cycle. The total number of Hamilton cycles of $G$ denoted by $T(H)$. Two Hamilton cycles $H_{1}$ and $H_{2}$ in $G$ are said to be edge disjoint if the edge sets $E\left(H_{1}\right)$ and $E\left(H_{2}\right)$ are disjoint.

Theorem. 7.1 For each positive integer $n \geq 3$, the odd permutation graph $G\left(S_{n}, B_{n}\right)$ is Hamiltonian.

Proof. Suppose $n \geq 3$ is a positive integer. Then, we construct the cycle $C=\left(f_{1}, g_{1}, f_{2}, g_{2}, \ldots, f_{\frac{n !}{2}}, g_{\frac{n !}{2}}, f_{1}\right)$ in $G\left(S_{n}, B_{n}\right)$. Here the cycle $C$ contains all the vertices of $G\left(S_{n}, B_{n}\right)$ exactly once, $C$ is a Hamilton cycle of $G\left(S_{n}, B_{n}\right)$. Hence $G\left(S_{n}, B_{n}\right)$ is Hamiltonian.

Theorem. 7.2 For each positive integer $n \geq 3$, the total number of Hamilton cycles in $G\left(S_{n}, B_{n}\right)$ is $T(H)=2\left(\frac{n !}{2}\right) !$.

Proof. From the Theorem [7.1], the cycle $C=\left(f_{1}, g_{1}, f_{2}, g_{2}, \ldots, f_{\frac{n}{2}}, g_{\frac{n !}{2}}, f_{1}\right)$ is a Hamilton cycle in $G\left(S_{n}, B_{n}\right)$. The number of arrangements of $\frac{n !}{2}$ odd permutations in $S_{n}$ are arranged between different fixed $\frac{n !}{2}$ even permutations from a collection of $n$ ! permutations is $\left(\frac{n !}{2}\right) !$, and vice versa, and hence each one of these arrangements is a cycle of length $n$ !, which is Hamilton cycle 
of the graph $G\left(S_{n}, B_{n}\right)$. From Theorem [6.2 ], the total number of Hamilton cycles in $G\left(S_{n}, B_{n}\right)$ is

$$
T(H)=\left(\frac{n !}{2}\right) !+\left(\frac{n !}{2}\right) !=2\left(\frac{n !}{2}\right) !
$$

Example. 7.3 The graph $G\left(S_{3}, B_{3}\right)$ contains 12 Hamilton cycles which are listed below.



$\left(f_{1}, g_{1}, f_{2}, g_{2}, f_{3}, g_{3}, f_{1}\right),\left(f_{1}, g_{1}, f_{2}, g_{3}, f_{3}, g_{2}, f_{1}\right),\left(f_{1}, g_{2}, f_{2}, g_{3}, f_{3}, g_{1}, f_{1}\right)$,

$\left(f_{1}, g_{3}, f_{2}, g_{2}, f_{3}, g_{1}, f_{1}\right),\left(f_{1}, g_{3}, f_{2}, g_{1}, f_{3}, g_{2}, f_{1}\right),\left(f_{1}, g_{2}, f_{2}, g_{1}, f_{3}, g_{3}, f_{1}\right)$,

$\left(g_{1}, f_{1}, g_{2}, f_{2}, g_{3}, f_{3}, g_{1}\right),\left(g_{1}, f_{2}, g_{2}, f_{3}, g_{3}, f_{1}, g_{1}\right),\left(g_{1}, f_{3}, g_{2}, f_{2}, g_{3}, f_{1}, g_{1}\right)$.

$\left(g_{1}, f_{3}, g_{2}, f_{2}, g_{3}, f_{1}, g_{1}\right),\left(g_{1}, f_{3}, g_{2}, f_{1}, g_{3}, f_{2}, g_{1}\right),\left(g_{1}, f_{2}, g_{2}, f_{1}, g_{3}, f_{3}, g_{1}\right)$

The Example [7.3] tells us that any two Hamilton cycles in the odd permutation graph are not edge disjoint. This immediate gives the following result.

Theorem. 7.4 The odd permutation graph $G\left(S_{n}, B_{n}\right)$ does not contain disjoint Hamilton cycles. That is, the total number of disjoint Hamilton cycles in $G\left(S_{n}, B_{n}\right)$ is zero.

Proof: Suppose $H_{1}, H_{2}, \ldots, H_{T(H)}$ be the total number of edge disjoint Hamilton cycles in the graph $G\left(S_{n}, B_{n}\right)$. Then, $E\left(H_{1}\right) \cap E\left(H_{2}\right) \cap \ldots \cap E\left(H_{T(H)}\right)=\phi$.

$\Rightarrow\left|E\left(H_{1}\right)\right|+\left|E\left(H_{2}\right)\right|+\ldots+\left|E\left(H_{T(H)}\right)\right|=\left|E\left(G\left(S_{n}, B_{n}\right)\right)\right|$.

$\Rightarrow T(H) n !=\left(\frac{n !}{2}\right)^{2}$, since $\left|E\left(H_{i}\right)\right|=n !, 1 \leq i \leq T(H)$. But by

the Theorem [7.2], we have $\left(2\left(\frac{n !}{2}\right) !\right) n !=\left(\frac{n !}{2}\right)^{2}$, which is

not true for each $n \geq 3$. So our assumption is wrong, and hence the total number of disjoint Hamilton cycles in $G\left(S_{n}, B_{n}\right)$ is zero.

\section{CONCLUSION}

In this paper, we have introduced new graph structures, called even and odd permutation graphs. An important outcome of this paper is that for all values of $n \geq 3$, these graphs are regular simple undirected graphs. Some other properties related to degree of vertices, enumeration of edges and cycles, and complete bipartite property has also been examined. It is our hope that this work would other foundation for further study of the theory of permutation graphs.

In our further study of permutation graphs, may be the following topics should be considered: (i) to find Clique number, (ii) to find Chromatic number, (iii) to obtained Domination parameters.

\section{ACKNOWLEDGMENT}

The authors express their sincere thanks to Prof.L.Nagamuni Reddy and Prof.S.Vijaya Kumar Varma for his suggestions during the preparation of this paper and the referee for his suggestions.

\section{REFERENCES}

[1] Dejter, I., Giudici, R.E.: On Unitary Cayley graphs, JCMCC, 18,121-124.

[2] Brrizbitia, P and Giudici, R.E. 1996, Counting pure kcycles in sequence of Cayley graphs, Discrete math., $149,11-18$.

[3] Madhavi, L and Maheswari, B.2009, Enumeration of Triangles and Hamilton Cycles in Quadratic residue Cayley graphas, Chamchuri Journal of Mathematics, 1,95-103.

[4] Madhavi, L and Maheswari, B. 2010, Enumeration of Hamilton cycles and triangles in Euler Totient Cayley graph, Graph Theory Notes of New York, LIX, 28-31.

[5] Chalapathi, T., Madhavi, L and Venkataramana, S. 2013 , Enumeration of Traingles in a Divisor Cayley Graph. Momona Ethiopian Journal of Science (MEJS). 5, 163173.

[6] Chalapathi, T., Kiran Kumar R.V.M.S.S. 2015, The Structural Properties of Even Free Graphs. International Journal of Computer Applications. 121, 19-21.

[7] Chartrand,G., Harary,F. 1967, Planner Permutaion Graphs, Ann. Inst. H. Poincare Sect. B(N, S) 3, 433-438.

[8] Harary F. 1969, Graph Theory, Addition-Wesley Publ. Comp., Regarding, Massachusett.

[9] Judson, T.W. 1994, Abstract Algebra, PWS Publishing Company, Boston .

[10] Alan Tucker. 2011, Applied Combinatorics, John Wiley and Sons Pte Ltd, Singapore.

[11] Bondy and J.A., Murty, U.S.R. 2013, Graph Theory. Ane Books-New Delhi. 